\title{
UNIQUE HAHN-BANACH THEOREMS FOR SPACES OF HOMOGENEOUS POLYNOMIALS
}

\author{
R. ARON, C. BOYD and Y. S. CHOI
}

(Received 16 March 1999; revised 8 November 2000)

\author{
Communicated by P. G. Dodds
}

\begin{abstract}
We investigate certain norm and continuity conditions that provide us with 'unique Hahn-Banach Theorems' from $\mathscr{P}\left({ }^{n} c_{0}\right)$ to $\mathscr{P}\left({ }^{n} \ell_{\infty}\right)$ and from $\mathscr{P}_{N}\left({ }^{n} E\right)$ to $\mathscr{P}_{N}\left({ }^{n} E^{\prime \prime}\right)$. We show that there is a unique norm-preserving extension for norm-attaining 2-homogeneous polynomials on complex $c_{0}$ to $\ell_{\infty}$ but there is no unique norm-preserving extension from $\mathscr{P}\left({ }^{3} c_{0}\right)$ to $\mathscr{P}\left({ }^{3} \ell_{\infty}\right)$.
\end{abstract}

2000 Mathematics subject classification: primary 46G25, 46A22.

\section{Introduction}

The problem of extending (continuous) homogeneous polynomials from a subspace of a Banach space to the entire space was first studied by the first author and Berner [1] in 1978. (The definition of homogeneous polynomial on a Banach space and other related concepts are reviewed below.) They showed that in contrast to linear functionals, extensions of homogeneous polynomials may not always exist. However, it was also shown that for all $n$, every $n$-homogeneous polynomial $P$ on a Banach space $E$ extends to an $n$-homogeneous polynomial $\tilde{P}$ on its bidual $E^{\prime \prime}$. It is this class of extensions which has received most attention to date (see $[1,4,5,8,9,13,17]$ ).

It was not until 1989 that Davie and Gamelin [5] showed that a 'true' Hahn-Banach extension theorem holds in this situation, by proving that $\|P\|_{E}=\|\tilde{P}\|_{E^{\prime \prime}}$. The purpose of this article is to examine the following question: Under what conditions do we have a unique extension for spaces of homogeneous polynomials? We will look at

Third author's research supported by grant No. 1999-2-102-003-5 from the Interdisciplinary Research Program of the KOSEF and by POSTECH/BSRI Special Fund.

(C) 2001 Australian Mathematical Society 0263-6115/2001 \$A2.00+0.00 
extending homogeneous polynomials from $c_{0}$ to $\ell_{\infty}$ in Section 2. In Section 3, we will show that in order to have a unique Hahn-Banach Theorem it is necessary not only that the norm of a homogeneous polynomial over $\ell_{\infty}$ be equal to its norm over $c_{0}$ but that the norms of all its derivatives at every point when taken over $\ell_{\infty}$ coincide with the norms of the corresponding polynomials when taken over $c_{0}$. Finally, in Section 4 , we examine norm-preserving extensions of nuclear polynomials from an M-ideal $E$ to its bidual.

We collect here some basic definitions which will be needed in the rest of the paper. Most of this material can be found, for example, in [7]. Given a Banach space $E$ we shall use $B_{E}$ to denote the closed unit ball of $E$. We say that $P: E \rightarrow K=\mathbb{R}$ or $\mathbb{C}$ is an $n$-homogeneous polynomial if there is a continuous $n$-linear form $A: E \times \cdots \times E \rightarrow K$ such that $P(x)=A(x, \ldots, x)$ for all $x \in E$. We shall use $\mathscr{P}\left({ }^{n} E\right)$ to denote the space of all $n$-homogeneous polynomials on $E$. Note that a 1-homogeneous polynomial is just a linear form. An application of the polarization formula yields the fact that there is a one-to-one correspondence between $n$-homogeneous polynomials $P$ and symmetric $n$-linear forms $\check{P}$ such that $P(x)=\check{P}(x, \ldots, x)$. The canonical extension $\tilde{P}: E^{\prime \prime} \rightarrow$ $K$ of an $n$-homogeneous polynomial $P: E \rightarrow K$ is given by means of the extension of the corresponding $n$-linear form $A$ : For an $n$-tuple $\left(z_{1}, \ldots, z_{n}\right) \in E^{\prime \prime} \times \cdots \times E^{\prime \prime}$, define $\tilde{A}: E^{\prime \prime} \times \cdots \times E^{\prime \prime} \rightarrow K$ by $\tilde{A}\left(z_{1}, \ldots, z_{n}\right)=\lim _{\alpha_{1}} \cdots \lim _{\alpha_{n}} A\left(x_{\alpha_{1}}, \ldots, x_{\alpha_{n}}\right)$, where each $\left(x_{\alpha_{i}}\right)$ is a net in $E$ which converges to $z_{j}$ in the weak topology. Although the definition of $\tilde{A}\left(z_{1}, \ldots, z_{n}\right)$ depends on the order in which one calculates the limits, the definition of the extended polynomial, $\tilde{P}(x) \equiv \tilde{A}(x, \ldots, x)$, is independent of the order used (see [7, Section 6.2] for further details). An $n$-homogeneous polynomial $P$ is said to be nuclear if there exists a bounded sequence $\left(\phi_{j}\right) \subset E^{\prime}$ and a sequence $\left(\lambda_{j}\right) \in \ell_{1}$ such that $P(x)=\sum_{j=1}^{\infty} \lambda_{j} \phi_{j}^{n}(x)$. Given a nuclear polynomial, we define its nuclear norm $\|P\|_{N} \equiv \inf \left\{\sum_{j=1}^{\infty}\left|\lambda_{j}\right|\left\|\phi_{j}\right\|^{n}: P(x)=\sum_{j=1}^{\infty} \lambda_{j} \phi_{j}^{n}(x)\right.$ all $x$ in $\left.E\right\}$. We use $\mathscr{P}_{N}\left({ }^{n} E\right)$ to denote the space of all $n$-homogeneous nuclear polynomials on $E$.

\section{Unique norm-preserving extensions from $c_{0}$ to $\ell_{\infty}$}

A Banach space $E$ is said to be an M-ideal in its bidual, $E^{\prime \prime}$, if $E^{\prime \prime \prime}$ is the $\ell_{1}$ sum of $E^{\prime}$ and $E^{\perp}=\left\{\phi \in E^{\prime \prime \prime}:\left.\phi\right|_{E} \equiv 0\right\}$; that is, every continuous linear functional $\phi$ on $E^{\prime \prime}$ can be written as

$$
\phi=\phi_{1}+\phi_{2}
$$

$\phi_{1} \in E^{\prime}, \phi_{2} \in E^{\perp}$, with

$$
\|\phi\|=\left\|\phi_{1}\right\|+\left\|\phi_{2}\right\| .
$$

It is immediate from this definition that when $E$ is an M-ideal in its bidual that every continuous linear functional on $E$ has a unique norm preserving extension to $E^{\prime \prime}$. 
In particular, since $c_{0}$ is an $\mathrm{M}$-ideal in $\ell_{\infty}$ (see [11]), every continuous linear functional on $c_{0}$ has a unique norm preserving extension to $\ell_{\infty}$. In this section we examine the extent to which this result carries over to $n$-homogeneous polynomials on $c_{0}$ when $n>1$. We shall distinguish between the real and complex cases.

In the real case for every $n>1$ the polynomial $P(x)=x_{1}^{n}-x_{1}^{n-2} \phi^{2}$, where $x=\left(x_{1}, x_{2}, \ldots\right), \phi \in c_{0}^{\perp}, 0<\|\phi\| \leq 1$, is a norm-preserving extension of $Q(x)=x_{1}^{n}$, $x=\left(x_{1}, x_{2}, \ldots\right)$, to $\ell_{\infty}$ which is different from $x_{1}^{n}$ on $\ell_{\infty}$. In particular, $x_{1}^{2}-\phi^{2}$ is a norm-preserving extension of $x_{1}^{2}$ to $\ell_{\infty}$.

In the remainder of this section, we shall study the more complicated complex case, with different results depending on the degree of the space of polynomials. We shall begin by considering homogeneous polynomials of degree at least three, and for this the following lemma will be useful.

LEMMA 1. Let $n \geq 3$ be a positive integer. Then $|1+w|^{n}+2|1-w|^{n-1} \leq 2^{n}$, for all $w \in \mathbb{C},|w|=1$.

Proof. Let $x=|1+w|$ and $y=|1-w|$. Then $0 \leq x \leq 2$ and $x^{2}+y^{2}=4$ (consider the triangle with vertices $1,-1$ and $w$ in the unit circle). Then

$$
|1+w|^{n}+2|1-w|^{n-1}=x^{n}+2\left(4-x^{2}\right)^{(n-1) / 2} .
$$

Hence it is enough to show that

$$
x^{n}+2\left(4-x^{2}\right)^{(n-1) / 2} \leq 2^{n}
$$

for $0 \leq x \leq 2$. By dividing both sides by $2^{n}$ and setting $t=x / 2$, it is equivalent to show that

$$
f(t)=t^{n}+\left(1-t^{2}\right)^{(n-1) / 2} \leq 1
$$

for $0 \leq t \leq 1$. Since

$$
t^{n}+\left(1-t^{2}\right)^{(n-1) / 2} \leq t^{3}+\left(1-t^{2}\right)
$$

for every $t \in[0,1]$ and since

$$
\max _{t \in[0,1]} t^{3}+\left(1-t^{2}\right)=1
$$

we conclude that

$$
f(t)=t^{n}+\left(1-t^{2}\right)^{(n-1) / 2} \leq 1
$$

Let $n \geq 3$ be an integer and consider the polynomial $P$ on $c_{0}$ defined by

$$
P(x)=\left(x_{1}+x_{2}\right)^{n},
$$

$x=\left(x_{1}, x_{2}, \ldots\right)$. Then $\|P\|=2^{n}=P\left(e_{1}+e_{2}\right)$. 
We define $\hat{P}_{1}$ on $\ell_{\infty}$ by

$$
\hat{P}_{1}(x)=\left(x_{1}+x_{2}\right)^{n},
$$

$x=\left(x_{1}, x_{2}, \ldots\right)$. It is clear that $\hat{P}_{1}$ is a norm-preserving extension of $P$.

Define $\hat{P}_{2} \in \mathscr{P}\left({ }^{n} \ell_{\infty}\right)$ by

$$
\hat{P}_{2}(x)=\left(x_{1}+x_{2}\right)^{n}+2\left(x_{1}-x_{2}\right)^{n-1} \phi(x),
$$

$x=\left(x_{1}, x_{2} \ldots,\right)$, where $\phi$ is a Banach limit functional on $\ell_{\infty}$ of norm at most 1 such that $\phi(1,-1,1, \ldots)=$,1 . Then clearly we have that $\left.\hat{P}_{2}\right|_{c_{0}}=P$ and since

$$
\hat{P}_{2}(1,-1,1,1, \ldots)=2^{n},
$$

we have that $\hat{P}_{1} \neq \hat{P}_{2}$. Fix $x=\left(x_{k}\right)_{k}$ in $B_{\ell_{\infty}}$. By the maximum modulus theorem we have

$$
\begin{aligned}
& \sup _{|z| \leq 1,|w| \leq 1}\left|\hat{P}_{2}\left(z, w, x_{3}, x_{4}, \ldots\right)\right| \\
& =\sup _{|z|=|w|=1}\left|\hat{P}_{2}\left(z, w, x_{3}, x_{4}, \ldots\right)\right| \leq \sup _{|z|=|w|=1}|z+w|^{n}+2|z-w|^{n-1} \\
& =\sup _{|z|=|w|=1} \frac{|z+w|^{n}}{|z|^{n}}+\frac{2|z-w|^{n-1}}{|z|^{n-1}}=\sup _{|z|=|w|=1}\left|1+\frac{w}{z}\right|^{n}+2\left|1-\frac{w}{z}\right|^{n-1} \\
& =\sup _{|w|=1}|1+w|^{n}+2|1-w|^{n-1} .
\end{aligned}
$$

Applying Lemma 1 we see that $\left\|\hat{P}_{2}\right\|$ is also equal to $2^{n}$. This shows that for each $n \geq 3$ on complex $c_{0}$ we are able to find an $n$-homogeneous polynomial which does not have unique norm-preserving extensions to $\ell_{\infty}$.

Let us turn to the case of 2-homogeneous polynomials on complex $c_{0}$. Since every $n$-homogeneous polynomial on $c_{0}$ is weakly continuous on bounded sets $[3,14]$ it follows from [6] that the monomials of degree $n$, with the square ordering, are a Schauder basis for $\mathscr{P}\left({ }^{n} c_{0}\right)$. Thus any $P$ in $\mathscr{P}\left({ }^{2} c_{0}\right)$ can be written as

$$
P(x)=\sum_{i=1}^{\infty}\left(\sum_{j=1}^{i} a_{i j} x_{i} x_{j}\right) .
$$

We shall say that $P$ is finite if there is an integer $n$ so that

$$
P(x)=\sum_{i=1}^{n}\left(\sum_{j=1}^{i} a_{i j} x_{i} x_{j}\right) .
$$

Proposition 2. A 2-homogeneous polynomial $P$ on complex $c_{0}$ is norm-attaining if and only if it is finite. 
Proof. If $P$ is finite we can regard it as a 2-homogeneous polynomial on $\ell_{\infty}^{n}$ and so it will attain its norm. Conversely suppose $P$ attains its norm at $x_{0}=\left(\lambda_{j}\right)_{j \in \mathbb{N}}$. Without loss of generality we shall assume that $\|P\|=1$. Let $J=\left\{j \in \mathbb{N}:\left|\lambda_{j}\right|=1\right\}$. Since $x_{0} \in c_{0}, J$ is finite. By change of variable and rearrangement of indices we may assume that $J=\{1,2, \ldots, n\}, \lambda_{1}=\lambda_{2}=\cdots=\lambda_{n}=1$ and that $\left|\lambda_{j}\right| \geq\left|\lambda_{j+1}\right|$ for all $j$. Given any $y$ in $B_{c_{0}}$ of the form

$$
y=(\underbrace{0, \ldots, 0}_{n \text {-times }}, y_{n+1}, y_{n+2}, \ldots),
$$

for every $\lambda \in \mathbb{C},|\lambda|=1-\left|\lambda_{n+1}\right|$, we have that

$$
\begin{aligned}
P\left(x_{0} \pm \lambda y\right)=\check{P}\left(x_{0} \pm \lambda y, x_{0} \pm \lambda y\right) & =\check{P}\left(x_{0}, x_{0}\right) \pm 2 \check{P}\left(x_{0}, \lambda y\right)+\check{P}(\lambda y, \lambda y) \\
& =1 \pm 2 \lambda \check{P}\left(x_{0}, y\right)+\lambda^{2} P(y),
\end{aligned}
$$

has modulus at most 1 , and thus

$$
\left|1+\lambda^{2} P(y)\right| \leq 1 .
$$

Choosing $\lambda$ so that $\lambda^{2} P(y)$ is purely imaginary we conclude that $P(y)=0$. In particular we get that $P\left(0,0, \ldots, 0, \lambda_{n+1}, \lambda_{n+2}, \ldots\right)=0$. We also have that $\breve{P}\left(x_{0}, y\right)$ is 0 for all $y$ of the above form. Taking $y=\left(0,0, \ldots, 0, \lambda_{n+1}, \lambda_{n+2}, \ldots\right)$ we see that $\check{P}\left((1,1, \ldots, 1,0,0, \ldots),\left(0,0, \ldots, 0, \lambda_{n+1}, \lambda_{n+2}, \ldots\right)\right)=0$ and therefore we have that $P(1,1, \ldots, 1,0,0, \ldots)=1$. Let $z_{1}=(\underbrace{1,1, \ldots, 1}_{n \text {-times }})$ and define $z_{2}, z_{3}, \ldots, z_{n}$ by

$$
\begin{aligned}
z_{2} & =(1,-n+1,1,1, \ldots, 1), \\
z_{3} & =(1,1,-n+1,1, \ldots, 1), \\
& \vdots \\
z_{n} & =(1,1,1, \ldots, 1,-n+1) .
\end{aligned}
$$

Since

$$
\left(x_{1}, x_{2}, \ldots, x_{n}\right)=\frac{1}{n}\left(x_{1}+x_{2}+\cdots+x_{n}\right) z_{1}+\frac{1}{n} \sum_{j=2}^{n}\left(x_{1}-x_{j}\right) z_{j},
$$

$\left\{z_{1}, z_{2}, \ldots, z_{n}\right\}$ forms a basis for $\mathbb{C}^{n}$. For $j=1, \ldots, n$, define $\tilde{z}_{j}$ in $c_{0}$ by $\tilde{z}_{j}=$ $\left(z_{j}, 0,0, \ldots\right)$. Repeating the argument given above we see that $\check{P}\left(\tilde{z}_{1}, y\right)=0$.

For any $\left(x_{1}, x_{2}, \ldots, x_{n}\right)$ in $\mathbb{C}^{n}$ we have

$$
\begin{aligned}
& P\left(x_{1}, x_{2}, \ldots, x_{n}, y_{n+1}, y_{n+2}, \ldots\right) \\
& \quad=P\left(x_{1}, x_{2}, \ldots, x_{n}, 0, \ldots\right)+\frac{2}{n} \sum_{j=2}^{n}\left(x_{1}-x_{j}\right) \check{P}\left(\tilde{z}_{j}, y\right) .
\end{aligned}
$$


Setting $2 / n \check{P}\left(\tilde{z}_{j}, \cdot\right)=\psi_{j} \in c_{0}^{\prime}$ we can write this as

$$
\begin{aligned}
& P\left(x_{1}, x_{2}, \ldots, x_{n}, y_{n+1}, y_{n+2}, \ldots\right) \\
& \quad=P\left(x_{1}, x_{2}, \ldots, x_{n}, 0, \ldots\right)+\sum_{j=2}^{n}\left(x_{1}-x_{j}\right) \psi_{j}(y) .
\end{aligned}
$$

We will show now that $\psi_{2} \equiv 0$. The same argument will work for any $j \geq 2$.

Set $x_{1}=1, x_{2}=e^{i \theta}$, and $x_{j}=1$ for $j>2$. Then

$$
P\left(1, e^{i \theta}, 1, \ldots, 1, y_{n+1}, y_{n+2}, \ldots\right)=P\left(1, e^{i \theta}, 1, \ldots, 1,0,0, \ldots\right)+\left(1-e^{i \theta}\right) \psi_{2}(y) .
$$

Since $\|P\|=1$ and since we can vary the argument of $y$ independent of $\theta$ so that

$$
\left|P\left(1, e^{i \theta}, 1, \ldots, 1,0, \ldots\right)\right|+\left|1-e^{i \theta}\right|\left|\psi_{2}(y)\right| \leq 1
$$

for all $y$ of norm at most 1 , we obtain that

$$
\left|P\left(1, e^{i \theta}, 1, \ldots, 1,0, \ldots\right)\right|+\left|1-e^{i \theta}\right||| \psi_{2}|| \leq 1 .
$$

Let $f(\theta)=\left|P\left(1, e^{i \theta}, 1, \ldots, 1,0, \ldots\right)\right|$ and $g(\theta)=\left|1-e^{i \theta}\right|$. Then we have

$$
\left\|\psi_{2}\right\| \leq \frac{1-f(\theta)}{g(\theta)}
$$

for all $\theta$. Since $P$ is a continuous 2 -homogeneous polynomial it is (complex) differentiable. Therefore we have that the functions

$$
\theta \in \mathbb{R} \rightarrow\left(\operatorname{Re} P\left(1, e^{i \theta}, 1, \ldots, 1,0, \ldots\right), \operatorname{Im} P\left(1, e^{i \theta}, 1, \ldots, 1,0, \ldots\right)\right) \in \mathbb{R}^{2}
$$
and

$$
(x, y) \in \mathbb{R}^{2} \backslash\{0\} \rightarrow \sqrt{x^{2}+y^{2}} \in \mathbb{R}
$$

are differentiable. Since $P\left(1, e^{i \theta}, 1, \ldots, 1,0, \ldots\right) \rightarrow 1$ as $\theta \rightarrow 0$ their composition, $f$, is also differentiable at $\theta=0$. Since $P$ has a local maximum on the unit sphere at $\theta=0$ we have $\lim _{\theta \rightarrow 0} f^{\prime}(\theta)=0$. Since $g(\theta)=[2(1-\cos \theta)]^{1 / 2}=2 \sin (\theta / 2)$, $\lim _{\theta \rightarrow 0} g^{\prime}(\theta)=1$. Applying L'Hôspital's rule we have $\left\|\psi_{2}\right\| \leq \lim _{\theta \rightarrow 0} f^{\prime}(\theta) / g^{\prime}(\theta)=0$. Thus $\psi_{j}$ is 0 for $j=2, \ldots, n$ and hence $P$ depends only on finitely many variables.

REMARK. The corresponding result for homogeneous polynomials on $c_{0}$ of degree greater than 2 fails. Lemma 1 can be used to show that the $k$-homogeneous polynomial

$$
P(x)=\left(x_{1}+x_{2}\right)^{k}+2\left(x_{1}-x_{2}\right)^{k-1} \sum_{j=3}^{\infty}\left(\frac{x_{j}}{2^{j}}\right),
$$

$k \geq 3$, attains it norm, yet is not finite. 
Let us now consider norm-preserving extensions of 2-homogeneous polynomials from complex $c_{0}$ to $\ell_{\infty}$. A straightforward adaptation of the argument given in Proposition 2 yields the following result.

PROPOSITION 3. Every 2-homogeneous norm-attaining polynomial on complex $c_{0}$ has a unique norm-preserving extension to $\ell_{\infty}$.

A Banach space $E$ with the property that every linear functional on $E$ has a unique norm preserving extension to $E^{\prime \prime}$ is said to be Hahn-Banach smooth (see [11, 16]). In [15] Smith and Sullivan introduce the weaker concept of weak HahnBanach smoothness by requiring that every norm attaining linear functional on $E$ has a unique norm preserving extension to $E^{\prime \prime}$. Thus we see that complex $c_{0}$ is 'weakly Hahn-Banach smooth' of degree 2 but not of degree 3 or higher.

The argument in Proposition 2 can be easily modified to show that every 2 homogeneous polynomial on $\ell_{\infty}^{k}$ has a unique norm-preserving extension to $\ell_{\infty}^{l}$, where $2 \leq k<l$. Using Lemma 1 we can show that for $n \geq 3$, the two polynomials $\hat{P}_{1}$ and $\hat{P}_{2}$ on $\ell_{\infty}^{l}$, defined by

$$
\hat{P}_{1}(x)=\left(x_{1}+x_{2}\right)^{n}
$$

and

$$
\hat{P}_{2}(x)=\left(x_{1}+x_{2}\right)^{n}+2\left(x_{1}-x_{2}\right)^{n-1} x_{3},
$$

are distinct norm preserving extensions of the polynomial

$$
P(x)=\left(x_{1}+x_{2}\right)^{n}
$$

on $\ell_{\infty}^{k}$.

We are unable to determine if every 2-homogeneous polynomial on $c_{0}$ has a unique norm-preserving extension to $\ell_{\infty}$. However, we do know that there are 2-homogeneous polynomials on $c_{0}$ which do not attain their norm and yet do have unique normpreserving extensions to $\ell_{\infty}$. For example, consider any polynomial $P$ on $c_{0}$ of the form

$$
P(x)=\sum_{n=1}^{\infty} a_{n} x_{n}^{2} .
$$

Let us suppose that this polynomial does not have a unique norm-preserving extension to $\ell_{\infty}$. We may suppose that $P$ has norm 1 and, by change of variable $x_{k} \rightarrow e^{i \theta_{k}} x_{k}$, that all of the $a_{n}$ 's are real and non-negative. Then there is $Q \in \mathscr{P}\left({ }^{2} \ell_{\infty}\right), Q$ vanishing on $c_{0}$, such that $\tilde{P}+Q$ is a norm-preserving extension of $P$ to $\ell_{\infty}$. Suppose that $\|Q\|>\delta>0$. Let $\epsilon=\delta / 20$ and choose an integer $n_{0}$ so that $\left\|\left.P\right|_{C^{n}}\right\|>1-\epsilon$ for all 
$n \geq n_{0}$. Let $u$ be a vector in $\mathbb{C}^{n}$ of the form $u= \pm e_{1} \pm e_{2} \pm \cdots \pm e_{n}$. Then for any $y$ in $B_{\ell_{x}}$ of the form $\left(0, \ldots, 0, y_{n+1}, y_{n+2}, \ldots\right)$ we have

$$
\tilde{P}(u \pm \lambda y)=P(u) \pm \lambda 2 \check{\tilde{P}}(u, y)+\lambda^{2} \tilde{P}(y) .
$$

The standard 'trick' (pick $\lambda$ so that $\lambda^{2} \tilde{P}(y)$ is real and positive) gives that $|\tilde{P}(y)| \leq \epsilon$. Replacing $\lambda$ by $i \lambda$ and adding to $(*)$ gives that

$$
P(u)+(1 \pm i) \lambda \check{\widetilde{P}}(u, y)
$$

has modulus at most 1 . Thus $|\check{\tilde{P}}(u, y)| \leq \epsilon$. We also have

$$
(\tilde{P}+Q)(u \pm \lambda y)=P(u) \pm 2 \lambda \check{P}(u, y) \pm 2 \lambda \check{Q}(u, y)+\lambda^{2} \tilde{P}(y)+\lambda^{2} Q(y) .
$$

Since $\|\tilde{P}+Q\| \leq 1$ we have that $|\check{\widetilde{P}}(u, y)+\check{Q}(u, y)| \leq \epsilon$ and $|\tilde{P}(y)+Q(y)| \leq \epsilon$. Hence $|\check{Q}(u, y)| \leq 2 \epsilon$ and $|Q(y)| \leq 2 \epsilon$.

We now make two observations:

1. Suppose that $B: \mathbb{R}^{n_{0}} \times \ell_{\infty} \rightarrow \mathbb{R}$ is a continuous bilinear form (where $\mathbb{R}^{n_{0}}$ has the supremum norm), such that for all choices $u= \pm e_{1} \pm e_{2} \pm \cdots \pm e_{n_{0}}$ and for all $y \in \ell_{\infty},\|y\| \leq 1$, we have $|B(u, y)| \leq \epsilon$. Then, in fact for all $x \in \mathbb{R}^{n_{0}},\|x\| \leq 1$ and for all $y \in \ell_{\infty},\|y\| \leq 1,|B(x, y)| \leq \epsilon$.

2. Suppose that $B: \mathbb{C}^{n_{0}} \times \ell_{\infty} \rightarrow C$ is a continuous bilinear form such that for all $x \in \mathbb{R}^{n_{0}},\|x\|_{\ell_{\alpha}^{n_{0}}} \leq 1$ and for all $y \in \ell_{\infty},\|y\| \leq 1,|B(x, y)| \leq \epsilon$. Then $\|B\| \leq 2 \epsilon$ (see for example [12]).

To conclude the argument, let $z$ be a point of $\ell_{\infty}$, of norm at most 1 . We can write $z$ as $z=x+y$, where $x$ is in $B_{\ell_{x}}^{n_{0}}$ and $y$ is of the above form. Then

$$
|Q(z)| \leq 2|\check{Q}(x, y)|+|Q(y)| \leq 10 \epsilon=\delta / 2
$$

which contradicts our assumption that $\|Q\|>\delta$.

\section{Characterizations of the canonical extension and norm-preserving extensions}

We have seen in the previous section that, at least for homogeneous polynomials of degree 3 or greater, the canonical norm-preserving extension from $\mathscr{P}\left({ }^{n} c_{0}\right)$ to $\mathscr{P}\left({ }^{n} \ell_{\infty}\right)$ is not unique. In this section we shall examine other properties that characterise the canonical extension from $\mathscr{P}\left({ }^{n} c_{0}\right)$ to $\mathscr{P}\left({ }^{n} \ell_{\infty}\right)$.

Let us begin with the following question. If $P$ is an $n$-homogeneous polynomial on $\ell_{\infty}$ and $\|P\|=\left\|\left.P\right|_{c_{0}}\right\|$, what can we conclude about $P$ ? 
Proposition 4. If $P \in \mathscr{P}\left({ }^{n} \ell_{\infty}\right)$ satisfies $\|P\|=\left\|\left.P\right|_{c_{0}}\right\|$, then $P$ is weak ${ }^{*}$ continuous on bounded sets at 0 .

ProOF. Given $y \in \ell_{\infty}$ and $k \in \mathbb{N}$ we define $\alpha_{k}(y)=\left(\alpha_{k}(y)_{i}\right)_{i=1}^{\infty} \in \ell_{\infty}$ by

$$
\alpha_{k}(y)_{i}= \begin{cases}y_{i} & \text { if } i \leq k \\ 0 & \text { if } i>k\end{cases}
$$

and $\omega_{k}(y)=\left(\omega_{k}(y)_{i}\right)_{i=1}^{\infty} \in \ell_{\infty}$ by

$$
\omega_{k}(y)_{i}= \begin{cases}0 & \text { if } i \leq k \\ y_{i} & \text { if } i>k\end{cases}
$$

We begin by showing that if $\|P\|=\left\|\left.P\right|_{c_{0}}\right\|$, then $\lim _{k \rightarrow \infty}\left\|\left.P\right|_{\omega_{k}\left(B_{t_{\infty}}\right)}\right\|=0$. Let us suppose that this condition does not hold. Then there is $C>0$, an increasing sequence of positive integers $\left(k_{n}\right)_{n \in \mathbb{N}}$ and a sequence of points $\left(y_{n}\right)_{n \in \mathbb{N}}$ in $B_{\ell_{\infty}}$ so that $\left|P\left(\omega_{k_{n}}\left(y_{n}\right)\right)\right|>C$. Choose $\epsilon>0$ so that $\epsilon<C^{2} /\left(4\left\|\left.P\right|_{c_{0}}\right\|\right)$. Since $\left(e_{n}\right)_{n \in \mathbb{N}}$ is a Schauder basis for $c_{0}$, we may choose $a \in B_{c_{0}}$ and a positive integer $n_{0}$ so that $\left|P\left(\alpha_{n_{0}}(a)\right)\right|>\left\|\left.P\right|_{c_{0}}\right\|-\epsilon$. Then $\left\{\alpha_{n_{0}}(a)+\lambda \omega_{k_{n_{0}}}\left(y_{n_{0}}\right):|\lambda| \leq 1\right\}$ is contained in $B_{\ell_{\infty}}$. Furthermore, by $[7$, Lemma $1.9(b)]$, we have that

$$
\begin{aligned}
\|P\|^{2} & \geq \sup _{|\lambda| \leq 1}\left|P\left(\alpha_{n_{0}}(a)+\lambda \omega_{k_{n_{0}}}\left(y_{n_{0}}\right)\right)\right|^{2} \geq\left|P\left(\alpha_{n_{0}}(a)\right)\right|^{2}+\left|P\left(\omega_{k_{n_{0}}}\left(y_{n_{0}}\right)\right)\right|^{2} \\
& >\left(\left\|\left.P\right|_{c_{0}}\right\|-\epsilon\right)^{2}+C^{2}>\left\|\left.P\right|_{c_{0}}\right\|^{2}+C^{2} / 2,
\end{aligned}
$$

a contradiction.

Now let us use this condition to show that $P$ is weak ${ }^{*}$ continuous on bounded sets at 0 . Suppose that $\left(x_{\beta}\right)_{\beta}$ is a bounded weak* null net in $\ell_{\infty}$, which we may suppose without loss of generality is contained in $B_{\ell_{\infty}}$. Given $\epsilon>0$ there is $n_{0} \in \mathbb{N}$ so that $\left\|\left.P\right|_{\omega_{n_{0}}\left(B_{\ell_{\infty}}\right)}\right\|<\epsilon / 2$. Then

$$
\left|P\left(x_{\beta}\right)\right|=\left|P\left(\alpha_{n_{0}}\left(x_{\beta}\right)+\omega_{n_{0}}\left(x_{\beta}\right)\right)\right| \leq \sum_{j=0}^{n}\left(\begin{array}{l}
n \\
j
\end{array}\right)\left|\check{P}\left(\alpha_{n_{0}}\left(x_{\beta}\right)\right)^{j}\left(\omega_{n_{0}}\left(B_{\varepsilon_{x}}\right)\right)^{n-j}\right| .
$$

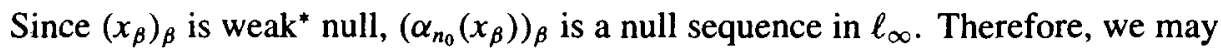
choose $\beta_{0}$ so that

$$
\left(\begin{array}{l}
n \\
j
\end{array}\right)\left|\check{P}\left(\alpha_{n_{0}}\left(x_{\beta}\right)\right)^{j}\left(\omega_{n_{0}}\left(B_{\ell_{\infty}}\right)\right)^{n-j}\right| \leq \frac{\epsilon}{2^{j+2}}
$$

for $\beta>\beta_{0}$ and all $j, 0<j \leq n$. This implies that $\left|P\left(x_{\beta}\right)\right| \leq \epsilon$ for $\beta>\beta_{0}$ and so $P$ is weak ${ }^{*}$ continuous on bounded sets at 0 . 
Using Proposition 4 we get the following 'unique Hahn-Banach Theorem' for the space of $n$-homogeneous polynomials on $c_{0}$ extended to $\ell_{\infty}$. The conditions in this theorem are analogous to those given in [17, Theorem 2], in which a characterization is given of when an analytic function on $E^{\prime \prime}$ is the canonical extension of an analytic function on $E$. Our condition is a weak* continuity condition on $P$ whereas Theorem 2 of [17] is a weak* continuity condition on the first derivative of $P$.

THEOREM 5. For $P \in \mathscr{P}\left({ }^{n} \ell_{\infty}\right)$ the following properties are equivalent:

(i) $P$ is the canonical extension of an $n$-homogeneous polynomial on $c_{0}$.

(ii) $P$ is weak ${ }^{*}$ continuous on bounded subsets.

$$
\left\|\frac{\hat{d}^{j} P}{j !}(x)\right\|=\left\|\left.\frac{\hat{d}^{j} P}{j !}(x)\right|_{c_{0}}\right\| \text { for every } x \text { in } \ell_{\infty} \text { and every integer } j, 1 \leq j \leq n .
$$

ProOF. It follows from the Littlewood-Bogdanowicz-Pełczyński Theorem (see [3, 14]) that every $n$-homogeneous polynomial $Q$ on $c_{0}$ is weakly continuous on bounded sets. Applying [2] we see that $Q$ is also weakly uniformly continuous on bounded sets and in particular on $B_{c_{0}}$. It therefore follows from Goldstine's Theorem that there is a unique weak* continuous extension, $\hat{Q}$, of $Q$ to $B_{\ell_{x}}$. Given $\xi \in B_{\ell_{x}}$ we apply [5] to get a net $\left(x_{\alpha}\right)_{\alpha}$ in $B_{c_{0}}$ such that for each positive integer $k$ and for each $R$ in $\mathscr{P}\left({ }^{k} c_{0}\right)$, $\left(R\left(x_{\alpha}\right)\right)_{\alpha}$ converges to $\tilde{R}(\xi)$. In particular, $\left(x_{\alpha}\right)$ converges weak* to $\xi$ and $\left(Q\left(x_{\alpha}\right)\right)_{\alpha}$ converges to $\tilde{Q}(\xi)$. Since $\hat{Q}$ is weak ${ }^{*}$ continuous on $B_{\ell_{\infty}},\left(Q\left(x_{\alpha}\right)\right)_{\alpha}$ converges to $\hat{Q}(\xi)$ and hence $\tilde{Q}=\hat{Q}$. Thus (i) and (ii) are equivalent.

Now suppose that (ii) holds. By the Polarization Formula it follows that $\left(\hat{d}^{j} P / j !\right)(x)$ is weak* continuous on bounded sets for every $x$ in $\ell_{\infty}$ and each positive integer $j$, $1 \leq j \leq n$. By Goldstine's Theorem, $B_{c_{0}}$ is weak* dense in $B_{\ell_{\infty}}$, from which (iii) follows.

If (iii) holds, then it follows from Proposition 4 that $\left(\hat{d}^{j} P / j !\right)(x)$ is weak continuous on bounded sets at 0 for every $x$ in $\ell_{\infty}$ and for every integer $j, 1 \leq j \leq n$. If $\left(x_{\alpha}\right)_{\alpha}$ is a bounded net which converges weak ${ }^{*}$ to $x$ in $\ell_{\infty}$, then $\left(x-x_{\alpha}\right)_{\alpha}$ is weak ${ }^{*}$ null. We now see that

$$
P(x)-P\left(x_{\alpha}\right)=\sum_{j=1}^{n}\left(\begin{array}{l}
n \\
j
\end{array}\right) \frac{\hat{d}^{j} P}{j !}(x)\left(x-x_{\alpha}\right)^{n-j}
$$

converges to 0 proving that $P$ is weak* continuous on bounded sets.

We have seen that there are homogeneous polynomials on $c_{0}$ which do not have unique norm preserving extensions to $\ell_{\infty}$. Motivated by Godefroy [10] we shall now give a criterion for a polynomial to have a unique norm preserving extension. In fact, this characterization holds for every Banach space $E$ such that $E^{\prime \prime}$ has the metric approximation property. (Recall that $E$ is said to have the metric approximation 
property if for every $\epsilon>0$ and every compact set $K \subset E$, there is a finite rank continuous linear operator $T: E \rightarrow E,\|T\| \leq 1$, such that $\|T x-x\|<\epsilon$ for every $x \in K$.) The linear version of the following theorem is due to Godefroy [10] (see also [11]).

THEOREM 6. Let $E$ be a Banach space such that $E^{\prime \prime}$ has the metric approximation property and $P \in \mathscr{P}\left({ }^{n} E\right)$ have norm 1 . Then the following are equivalent:

(i) $P$ has a unique norm preserving extension to $E^{\prime \prime}$.

(ii) If $\left(P_{\alpha}\right)_{\alpha}$ is a net in $B_{\left.\mathscr{P}^{(}{ }^{n} E\right)}$ which converges pointwise to $P$, then $\left(\tilde{P}_{\alpha}(x)\right)_{\alpha}$ converges to $\tilde{P}(x)$ for every $x$ in $E^{\prime \prime}$.

PROOF. Suppose that (i) holds and that $\left(P_{\alpha}\right)_{\alpha}$ is a net in $B_{\mathscr{P}\left({ }^{n} E\right)}$ which converges pointwise to $P$. Since $B_{\left.\mathscr{P}^{(}{ }^{\prime \prime} E^{\prime \prime}\right)}$ is compact for the compact-open topology, for every subnet $\left(P_{\beta}\right)_{\beta}$ of $\left(P_{\alpha}\right)_{\alpha}$ we can find $Q \in B_{\left.\mathscr{P}^{(n} E^{\prime \prime}\right)}$ and a subnet $\left(P_{\gamma}\right)_{\gamma}$ so that $\tilde{P}_{\gamma}(x)$ converges to $Q(x)$ for every $x$ in $E^{\prime \prime}$. Since $\left(\tilde{P}_{\gamma}\right)_{\gamma}$ is in $B_{\left.\mathscr{P}^{(n} E^{\prime \prime}\right)}$ and $\left(P_{\gamma}\right)_{\gamma}$ converges pointwise to $P, Q$ is a norm preserving extension of $P$ and therefore by our assumption $Q$ must be equal to $\tilde{P}$. In particular, $\left(\tilde{P}_{\alpha}(x)\right)_{\alpha}$ converges to $\tilde{P}(x)$ for all $x$ in $B_{E^{\prime \prime}}$ and so (ii) holds.

Conversely, suppose that (ii) holds and that $\hat{P} \in \mathscr{P}\left({ }^{n} E^{\prime \prime}\right)$ is a norm preserving extension of $P$. We may write $\hat{P}$ as $\hat{P}=\tilde{P}+Q$ where $Q \in\left\{R \in \mathscr{P}\left({ }^{n} E^{\prime \prime}\right):\left.R\right|_{E} \equiv 0\right\}$. Since $E^{\prime \prime}$ has the metric approximation property, using [4, Theorem 4.4] we can find a net $\left(P_{\alpha}\right)_{\alpha}$ in $B_{\left.\mathcal{S}^{(n}{ }^{n} E\right)}$ so that $\left(\tilde{P}_{\alpha}(x)\right)_{\alpha}$ converges to $\hat{P}(x)$ for every $x$ in $E^{\prime \prime}$. Clearly $\left(P_{\alpha}\right)_{\alpha}$ converges pointwise to $P$ on $B_{\left.\mathscr{P}^{(} E\right)}$. By our assumption $\left(\tilde{P}_{\alpha}(x)\right)$ converges to $\tilde{P}(x)$ for all $x$ in $E^{\prime \prime}$ and so $\hat{P}=\tilde{P}$.

The canonical extension $P \rightarrow \tilde{P}$ may be viewed as an isometry from $\mathscr{P}\left({ }^{n} E\right)$ into $\mathscr{P}\left({ }^{n} E^{\prime \prime}\right)$. Condition (ii) of Theorem 6 may be regarded as saying that the restriction of this function to $B_{\left.\mathscr{P}^{(n} E\right)}$ is pointwise-to-pointwise continuous at $P$.

\section{Unique norm-preserving extensions of nuclear polynomials}

In this section, the Banach space $E$ will be either real or complex, and we will examine the question of unique norm-preserving extensions of nuclear polynomials from a $E$ to $E^{\prime \prime}$. Let us begin with the observation that the canonical extension is a norm preserving extension from $\left(P_{N}\left({ }^{n} E\right),\|\cdot\|_{N}\right)$ to $\left(P_{N}\left({ }^{n} E^{\prime \prime}\right),\|\cdot\|_{N}\right)$. To show this, given any $P$ in $\left(P_{N}\left({ }^{n} E\right),\|\cdot\|_{N}\right)$ and $\epsilon>0$, choose a representation $\sum_{k=1}^{\infty} \lambda_{k} \phi_{k}^{n}$ of $P$ so that $\sum_{k=1}^{\infty}\left|\lambda_{k}\right|\left\|\phi_{k}\right\|^{n} \leq\|P\|_{N}+\epsilon$. Since $\sum_{k=1}^{\infty} \lambda_{k} \phi_{k}^{n}$ is a representation of the canonical extension $\tilde{P}$, we have that

$$
\|\tilde{P}\|_{N} \leq \sum_{k=1}^{\infty}\left|\lambda_{k}\right|\left\|\phi_{k}\right\|^{n} \leq\|P\|_{N}+\epsilon .
$$


Hence $\|\tilde{P}\|_{N} \leq\|P\|_{N}$. Conversely, we can choose a representation $\sum_{k=1}^{\infty} \mu_{k} \psi_{k}^{n}$ of $\tilde{P}$ so that $\sum_{k=1}^{\infty}\left|\mu_{k}\right|\left\|\psi_{k}\right\|^{n} \leq\|\tilde{P}\|_{N}+\epsilon$, where $\psi_{k} \in E^{\prime \prime \prime}$. Then we have

$$
\|\tilde{P}\|_{N}+\epsilon \geq \sum_{k=1}^{\infty}\left|\mu_{k}\right|\left\|\psi_{k}\right\|^{n} \geq \sum_{k=1}^{\infty}\left|\mu_{k}\right|\left\|\left.\psi_{k}\right|_{E}\right\|^{n} \geq\|P\|_{N}
$$

and therefore $\|\tilde{P}\|_{N} \geq\|P\|_{N}$.

The following is a partial answer to the question: When do we have a unique norm-preserving extension from $\mathscr{P}_{N}\left({ }^{n} E\right)$ to $\mathscr{P}_{N}\left({ }^{n} E^{\prime \prime}\right)$ ?

Proposition 7. Let E be a Banach space which is an M-ideal in its bidual. Then the canonical extension is the unique norm preserving extension from $P_{N}\left({ }^{n} E\right)$ to $P_{N}\left({ }^{n} E^{\prime \prime}\right)$.

Proof. Let $Q$ be an extension of $P$ which is not equal to $\tilde{P}$. We will show that $\|Q\|_{N}>\|P\|_{N}$. Since $Q \neq \tilde{P}$, there is $y \in E^{\prime \prime},\|y\|=1$, so that

$$
|Q(y)-\tilde{P}(y)|=\delta>0 .
$$

Choose a representation $\sum_{k=1}^{\infty} \lambda_{k} \phi_{k}^{n}$ of $Q$ so that $\sum_{k=1}^{\infty}\left|\lambda_{k}\right|\left\|\phi_{k}\right\|^{n} \leq\|Q\|_{N}+\delta / 2$, where each $\phi_{k} \in E^{\prime \prime \prime}$. Then

$$
\begin{aligned}
\delta=|Q(y)-\tilde{P}(y)| & =\left|\sum_{k=1}^{\infty} \lambda_{k} \phi_{k}(y)^{n}-\sum_{k=1}^{\infty} \lambda_{k} y\left(\left.\phi_{k}\right|_{E}\right)^{n}\right| \\
& \leq \sum_{k=1}^{\infty}\left|\lambda_{k}\right|\left|\phi_{k}(y)^{n}-y\left(\left.\phi_{k}\right|_{E}\right)^{n}\right| .
\end{aligned}
$$

Since $E$ is an M-ideal in $E^{\prime \prime}$ we have $\phi_{k}=\left.\phi_{k}\right|_{E}+\phi_{k}^{\perp}$, with $\phi_{k}^{\perp}$ in $E^{\perp}$ and $\left\|\phi_{k}\right\|=$ $\left\|\left.\phi_{k}\right|_{E}\right\|+\left\|\phi_{k}^{\perp}\right\|$. Therefore,

$$
\begin{aligned}
\delta & \leq \sum_{k=1}^{\infty}\left|\lambda_{k}\right|\left|\left(y\left(\left.\phi_{k}\right|_{E}\right)+\phi_{k}^{\perp}(y)\right)^{n}-y\left(\left.\phi_{k}\right|_{E}\right)^{n}\right| \\
& \leq \sum_{k=1}^{\infty}\left|\lambda_{k}\right|\left(\sum_{j=1}^{n}\left(\begin{array}{c}
n \\
j
\end{array}\right)\left\|\left.\phi_{k}\right|_{E}\right\|^{n-j}\left\|\phi_{k}^{\perp}\right\|^{j}\right) \\
& =\sum_{k=1}^{\infty}\left|\lambda_{k}\right|\left[\left(\left\|\left.\phi_{k}\right|_{E}\right\|+\left\|\phi_{k}^{\perp}\right\|\right)^{n}-\left\|\left.\phi_{k}\right|_{E}\right\|^{n}\right] \\
& =\sum_{k=1}^{\infty}\left|\lambda_{k}\right|\left\|\phi_{k}\right\|^{n}-\sum_{k=1}^{\infty}\left|\lambda_{k}\right|\left\|\left.\phi_{k}\right|_{E}\right\|^{n} \leq\|Q\|_{N}+\delta / 2-\|\tilde{P}\|_{N} .
\end{aligned}
$$


This means we have that

$$
\|Q\|_{N}-\|\tilde{P}\|_{N} \geq \delta / 2>0
$$

which completes the proof.

In particular, for each $n \in \mathbb{N}$, each nuclear polynomial on $c_{0}$ has a unique nuclear norm preserving extension to $\ell_{\infty}$.

\section{Acknowledgement}

The initial work on this paper was done in 1995-96 while the second author was Visiting Assistant Professor at Pohang University of Science and Technology. He wishes to thank the Department of Mathematics at that university for their hospitality. The authors would also like to thank the referee for a number of suggestions to a previous version of this paper.

\section{References}

[1] R. M. Aron and P. Berner, 'A Hahn-Banach extension theorem for analytic mappings', Bull. Math. Soc. France 106 (1978), 3-24.

[2] R. M. Aron, C. Herves and M. Valdivia, 'Weakly continuous mappings on Banach spaces', J. Funct. Anal. 52 (1983), 198-204.

[3] W. Bogdanowicz, 'On the weak continuity of the polynomial functionals on the space $c_{0}$ ', Bull. Acad. Polon. Sci. Sér. Math. Astronom. Phys. 5 (1957), 243-246 (in Russian).

[4] T. K. Carne, B. Cole and T. W. Gamelin, 'A uniform algebra of analytic functions on a Banach space', Trans. Amer. Math. Soc. 314 (1989), 639-659.

[5] A. M. Davie and T. W. Gamelin, 'A theorem on polynomial-star approximation', Proc. Amer. Math. Soc. 106 (1989), 351-356.

[6] V. Dimant and S. Dineen, 'Banach subspaces of spaces of holomorphic functions and related topics', Math. Scand. 83 (1998), 142-160.

[7] S. Dineen, Complex analysis on infinite dimensional spaces, Monographs in Math. (Springer, London, 1999).

[8] S. Dineen and R. Timoney, 'Complex geodesics on convex domains', in: Progress in Functional Analysis (ed. K. Bierstedt el al.), Math. Studies 170 (North-Holland, Amsterdam, 1992) pp. 333365.

[9] P. Galindo, D. García, M. Maestre and J. Mujica, 'Extension of multilinear mappings on Banach spaces', Studia Math. 108 (1994), 55-76.

[10] G. Godefroy, 'Points de Namioka, espaces normante, applications à la Théorie isométrique de la dualité', Israel J. Math. 38 (1981), 209-220.

[11] P. Harmand, D. Werner and W. Werner, M-ideal in Banach spaces and Banach algebras, Lecture Notes in Math. 1547 (Springer, Berlin, 1993).

[12] P. Kirwan, Complexification of multilinear and polynomial mappings on normed spaces (Ph.D. Thesis, National University of Ireland, Galway, 1997). 
[13] M. Lindström and R. Ryan, 'Applications of ultraproducts to infinite dimensional holomorphy', Math. Scand. 71 (1992), 229-242.

[14] A. Pełczyński, 'A property of multilinear operations', Studia Math. 16 (1957), 173-182.

[15] M. A. Smith and F. Sullivan, 'Extremely smooth Banach spaces', in: Banach spaces of analytic functions, Proc. Conf. Kent, Ohio 1976 (eds. J. Baker, C. Cleaver and J. Diestel), Lecture Notes in Math. 604 (Springer, Berlin, 1977) pp. 125-137.

[16] F. Sullivan, 'Geometric properties determined by higher duals of a Banach space', Illinois J. Math. 21 (1977), 315-331.

[17] I. Zalduendo, 'A canonical extension for analytic functions on Banach spaces', Trans. Amer. Math. Soc. 320 (1990), 747-763.

Department of Mathematics

Kent State University

Kent

Ohio 44242

USA

e-mail: aron@mcs.kent.edu
Department of Mathematics University College Dublin

Belfield

Dublin 4

Ireland

e-mail: Christopher.Boyd@ucd.ie

\author{
Department of Mathematics \\ Pohang University of Science and Technology \\ Pohang 790 \\ South Korea \\ e-mail: mathchoi@posmath.postech.ac.kr
}

